OFD or a metaphyseal fibrous defect/non-ossifying fibroma - the most likely diagnoses for tumour sited in the cortical bone. A haematoxylin and eosin-stained section of the needle core biopsy specimen showed the characteristic features of a chondroblastoma, despite the fact that the vast majority of chondroblastomas are intramedullary epiphyseal neoplasms. Many of the lesional cells were strongly and diffusely positive for CKs (extensively positive for MNF116, AE1/AE3, CAM5.2, CK8 and CK18 and more focally positive for CK19) (Figure 2).

Another diagnosis that should be considered in a case of an unusual $\mathrm{CK}+$ skeletal neoplasm is an extraaxial chordoma/chordoma periphericum, a neoplasm which also expresses CKs, specifically CK19. ${ }^{6}$ However, our $\mathrm{CK}+$ intracortical neoplasm was negative for brachyury, a transcription factor which is crucial for notochordal development. We have recently found this molecule to be expressed in $100 \%$ of 52 axial chordomas analysed and not in over 300 other tumours apart from one intracortical tibial neoplasm. ${ }^{2,3}$ This brachyury-positive extra-axial neoplasm exhibited characteristic, although not typical, morphology and typical immunohistochemistry (CK19, S100 and brachyury) of a chordoma. However, as it was not sited in the spinal column, we propose that it is more appropriate to classify such a neoplasm as one showing notochordal differentiation rather than a chordoma. Chordomas are considered to arise from notochordal remnants and it is unlikely that ectopic notochordal remnants account for the origin of this tumour.

K Bousdras
${ }^{1}$
P O'Donnell
S Vujovic $^{3}$
S Henderson
C Boshoff
A M Flanagan
1,4
Departments of ${ }^{1}$ Histopathology and
${ }^{2}$ Radiology, Royal National Orthopaedic
Hospital, Stanmore,
${ }^{3}$ Wolfson Institute for Biomedical Research,
University College London, London and
${ }^{4}$ Institute of Orthopaedics and Musculoskeletal Science,
University College London, Stanmore, UK

1. Henderson SR, Guiliano D, Presneau N et al. A molecular map of mesenchymal tumors. Genome Biol. 2005; 6; R76.

2. Vujovic S, Henderson SR, Presneau N et al. Brachyury, a crucial regulator of notochordal development, is a novel biomarker for chordomas. J. Pathol. 2006; 209; 157-165.

3. O'Donnell P, Tirabosco R, Vujovic S et al. Diagnosing an extraaxial chordoma of the proximal tibia with the help of brachyury, a molecule required for notochordal differentiation. Skeletal Radiol. 2007; 36; 59-65.

4. Edel G, Ueda Y, Nakanishi J et al. Chondroblastoma of bone. Virchows Arch. 1992; 421; 355-366.

5. Semmelink HJF, Pruszczynski M, Tilburg AWV, Smetds F, Raemaekers FCS. Cytokeratin expression in chondroblastomas. Histopathology 1990; 16; 257-263.

6. Nielsen GP, Mangham DC, Grimer RJ, Rosenberg AE. Chordoma periphericum: a case report. Am. J. Surg. Pathol. 2001; 25; 263267.

\section{Kinetic topographical heterogeneity in follicular thyroid neoplasms and growth patterns}

DOI: 10.1111/j.1365-2559.2007.02778.x

Sir: Several markers have been tried to help to differentiate prognostically relevant subgroups of follicular thyroid neoplasms; one of the latest was recently published in Histopathology. ${ }^{1}$ This paper highlights the proliferation profiles (differences in MCM2 and Ki67 expression) and the topographical distribution of these markers in follicular thyroid neoplasms diagnosed by World Health Organization criteria. $^{2}$ The immunoexpression was scored on the basis of positive nuclei staining homogeneously for MCM2 or Ki67 and assessing tumour cells in the peripheral and central part of each tumour. The authors reported no correlation between MCM2 and Ki67 (Spearman's correlation coefficient $r=0.149$ ) and MCM2 expression predominated in peripheral tumour cells in most of the minimally invasive follicular thyroid carcinomas (FTC), whereas it was diffuse with no apparent topographical differences in many follicular thyroid adenomas (FTA). ${ }^{1}$

I have topographically analysed the kinetic features [proliferation by Ki67 index and apoptosis by in situ end labelling (ISEL) or ISEL index] of follicular thyroid neoplasms (43 FTA, 76 FTC-including 28 minimally invasive and 48 widely invasive - and 27 anaplastic carcinomas) and have obtained similar results. Apart from the diagnostic applicability, these results provide insight into the basis of the known architectural patterns of thyroid tumours.

The Ki67/ISEL indices provide kinetic advantage in the internal compartments of benign lesions and in the peripheral compartments of malignant lesions (Table 1), due to the statistically significant decrease of ISEL indices in internal compartments in adenomas $(5.3 \pm 7.8 \%$ versus $1.3 \pm 2.4 \% ; P=0.0213)$ and in peripheral compartments in carcinomas $(1.4 \pm 1.7 \%$ versus $2.3 \pm 5.5 \% ; P=0.0474)$. Similarly, in the 
Table 1. Ki67 and ISEL indices by topographical compartments in follicular thyroid proliferative lesions

\begin{tabular}{|c|c|c|c|c|c|c|}
\hline & \multicolumn{3}{|c|}{ Peripheral compartment } & \multicolumn{3}{|c|}{ Internal compartment } \\
\hline & $\begin{array}{l}\mathrm{Ki} 67(\%) \\
A v \pm S D\end{array}$ & $\begin{array}{l}\text { ISEL (\%) } \\
A v \pm S D\end{array}$ & $\begin{array}{l}\mathrm{Ki} 67 / \mathrm{ISEL} \\
\text { index (\%) }\end{array}$ & $\begin{array}{l}\mathrm{Ki} 67(\%) \\
A v \pm S D\end{array}$ & $\begin{array}{l}\text { ISEL (\%) } \\
A v \pm S D\end{array}$ & $\begin{array}{l}\text { Ki67/ISEL } \\
\text { index (\%) }\end{array}$ \\
\hline FTA & $4.9 \pm 3.5$ & $5.3 \pm 7.8$ & $0.9 \pm 0.7$ & $6.8 \pm 7.5$ & $1.3 \pm 2.4$ & $5.2 \pm 5.8$ \\
\hline MI-FTC & $8.6 \pm 13$ & $5.8 \pm 12$ & $1.5 \pm 2.2$ & $2.9 \pm 3$ & $4.3 \pm 10$ & $0.7 \pm 0.7$ \\
\hline WI-FTC & $7.6 \pm 8.8$ & $1.4 \pm 1.7$ & $5.4 \pm 6.3$ & $3.5 \pm 4.8$ & $2.3 \pm 5.5$ & $1.5 \pm 2.1$ \\
\hline ATC & $53 \pm 50$ & $9 \pm 15$ & $5.9 \pm 5.6$ & $36 \pm 32$ & $1 \pm 1$ & $36.0 \pm 32.0$ \\
\hline
\end{tabular}

FTA, Follicular thyroid adenoma; MI-FTC, minimally invasive follicular thyroid carcinoma; WI-FTC, widely invasive follicular thyroid carcinoma; ATC, anaplastic thyroid carcinoma; ISEL, in situ end labelling; Av, average.

majority of minimally invasive FTCs, Mar et al. found more MCM2+ cells in the peripheral invasive areas compared with central areas; the MCM2+ cell rate appeared to be similar to that of FTA. ${ }^{1}$ The results suggest that tumour cells in the invasive areas of minimally invasive FTC have increased proliferative activity regardless of the presence of capsular invasion, vascular invasion or both. In our series, benign lesions (FTA) and low-grade malignancies (minimally invasive FTC) showed higher rates of apoptosis in peripheral compared with internal compartments, whereas highgrade malignancies (widely invasive FTC and anaplastic thyroid carcinomas) had the inverse relationship (Table 1).

Tumours can be divided into two topographical compartments (superficial and deep in luminal organs equivalent to internal and peripheral in solid organs, respectively) with different cell kinetic and genetic profiles. $^{3-5}$ Superficial/internal compartments are predominantly proliferative with a lower incidence of genetic abnormalities, while in deep/peripheral compartments invasion predominates and cells accumulate more genetic alterations. In general, proliferation and active infiltration would not be expected simultaneously in the same topographical compartment, which may in part explain capsule development in solid organ neoplasms. The kinetic profile provides a basis for disciminating benign from malignant lesions and an explanation of why benign lesions are well circumscribed and encapsulated. Inverse and opposite proliferation/apoptosis correlations characterize benign and malignant tumours, the kinetic advantage predominating in internal compartments of benign lesions and in peripheral compartments of malignant lesions. Benign lesions demonstrate an inverse proliferation-apoptosis correlation (increasing apoptosis and decreasing proliferation) in the peripheral compartments and a direct correlation in internal compart- ments. The opposite profile is seen in carcinomas, confirming the kinetic equivalence of superficial-internal and deep-peripheral compartments of neoplasms from luminal and solid organs. ${ }^{3-5}$ Cell kinetics (proliferation and apoptosis) represent the basic mechanism leading to clonal expansion and tumour growth ${ }^{6-11}$ and contribute to the theory of tumour cell topographical segregation. ${ }^{3-5,12,13}$ High proliferation rates in internal compartments of FTA support this theory. Significantly higher apoptosis rates in peripheral than in internal compartments of benign lesions suggest the accumulation of genetic mutations.

Benign lesions (FTA) and low-grade malignancies (minimally invasive FTC) show higher rates of apoptosis in peripheral compared with internal compartments, whereas high-grade malignancies (widely invasive FTC and anaplastic thyroid carcinoma) reveal the inverse relationship. Whether this indicates progression of the peripheral tumour cells in high-grade follicular neoplasms remains to be determined. However, evidence can be found in urothelial carcinoma of the bladder and adrenal pheochromocytoma. ${ }^{3-5}$ Highgrade neoplasms show high rates of apoptosis, which should be the result of the accumulation of genetic mutations reaching levels lethal to cells. ${ }^{7-9}$ Follicular tumour progression correlates with up-regulation of proliferation and down-regulation of apoptosis in peripheral compartments and suggests survival and replication of genetically damaged cells leading to the accumulation of genetic mutations: ${ }^{3-5,12,13}$ high cell proliferation would transfer mutations to daughter cells, whereas reduced apoptosis would allow cells carrying mutations to complete the cell cycle.

In conclusion, differential cell kinetics in follicular thyroid neoplasms contributes to a topographical segregation of tumour cells and the growth pattern (encapsulation and invasiveness at the tumour periphery). 


\section{S J Diaz-Cano}

Department of Histopathology, King's College Hospital and King's College London School of Medicine, London, UK

1. Mar KC, Eimoto T, Nagaya S, Tateyama H. Cell proliferation marker MCM2, but not Ki67, is helpful for distinguishing between minimally invasive follicular carcinoma and follicular adenoma of the thyroid. Histopathology 2006; 48; 801-807.

2. Lloyd R, DeLellis R, Heitz P, Eng C. Pathology and genetics of tumours of endocrine organs. Geneva: WHO Press 2004.

3. Diaz-Cano SJ, Blanes A, Rubio J, Matilla A, Wolfe HJ. Molecular evolution and intratumor heterogeneity by topographic compartments in muscle-invasive transitional cell carcinoma of the urinary bladder. Lab. Invest. 2000; 80; 279-289.

4. Blanes A, Rubio J, Martinez A, Wolfe HJ, Diaz-Cano SJ. Kinetic profiles by topographic compartments in muscle-invasive transitional cell carcinomas of the bladder: role of TP53 and NF1 genes. Am. J. Clin. Pathol. 2002; 118; 93-100.

5. Blanes A, Sanchez-Carrillo JJ, Diaz-Cano SJ. Topographic molecular profile of pheochromocytomas: role of somatic downregulation of mismatch repair. J. Clin. Endocrinol. Metab. 2006; 91; 1150-1158.

6. Diaz-Cano SJ. Adrenal medullary proliferation and clonality. In: 87th Annual Meeting of the US and Canadian Academy of Pathology; 28 February to 6 March 1998; Boston, MA, USA. 1998.

7. Diaz-Cano SJ, Blanes A, Wolfe HJ. PCR techniques for clonality assays. Diagn. Mol. Pathol. 2001; 10; 24-33.

8. Diaz-Cano SJ. Designing a molecular analysis of clonality in tumours. J. Pathol. 2000; 191; 343-344.

9. Diaz-Cano SJ. Clonality studies in the analysis of adrenal medullary proliferations. Application principles and limitations. Endocr. Pathol. 1998; 9; 301-316.

10. Diaz-Cano SJ. MAP kinase common pathway in thyroid carcinomas of follicular differentiation. J. Pathol. 2006; 209; 298-306.

11. Pomerance M, Quillard J, Chantoux F, Young J, Blondeau JP. High-level expression, activation, and subcellular localization of p38-MAP kinase in thyroid neoplasms. J. Pathol. 2006; 209; 298-306.

12. Diaz-Cano SJ, de Miguel M, Blanes A, Tashjian R, Galera H, Wolfe HJ. Clonality as expression of distinctive cell kinetics patterns in nodular hyperplasias and adenomas of the adrenal cortex. Am. J. Pathol. 2000; 156; 311-319.

13. Diaz-Cano SJ, de Miguel M, Blanes A, Tashjian R, Galera H, Wolfe HJ. Clonal patterns in phaeochromocytomas and MEN-2A adrenal medullary hyperplasias: histological and kinetic correlates. J. Pathol. 2000; 192; 221-228.

\section{Co-secretion of testosterone and cortisol by a functional adrenocortical oncocytoma}

DOI: $10.1111 /$ j.1365-2559.2007.02780.x

Sir: Oncocytic adrenocortical neoplasms are rare and most are non-functional. ${ }^{1}$ To date, we are aware of only three cases of adrenocortical oncocytic tumours in the English literature reported to have been hormonally active. ${ }^{2-4}$ We describe a further case, which was found to secrete testosterone and cortisol. Only one other virilizing adrenal oncocytoma has been reported, and in that case a documented increase in serum testosterone was not recorded.

A 58-year-old postmenopausal lady was referred to the Diabetes Centre due to poor glycaemic control and problematic hypertension despite maximal oral hypoglycaemic treatment and the use of two antihypertensive agents. She was observed to have male pattern hair loss and testosterone levels returned at $5.7 \mathrm{nmol} / \mathrm{l}$ (normal range <3). An adrenal source was suspected as both androstenedione and dihydroepiandrostenedione sulphate (DHEAS) $[15.5 \mathrm{nmol} / \mathrm{l}$ (normal range 3-12) and $8.5 \mu \mathrm{mol} / \mathrm{l}$ (normal range 1-8.5), respectively] were elevated. A computed tomography scan revealed an $87 \times 88 \mathrm{~mm}$ mass at the upper pole of the right kidney arising from the body of the right adrenal gland, which demonstrated homogeneous enhancement following intravenous contrast. Preoperatively a 1-mg overnight low-dose dexamethasone suppression test confirmed low-grade Cushing's syndrome (baseline cortisol $606 \mathrm{nmol} / \mathrm{l}$, normal range 155-605 nmol/l; post $1 \mathrm{mg}$ dexamethasone cortisol $348 \mathrm{nmol} / \mathrm{l}$, normal value $<50 \mathrm{nmol} / \mathrm{l})$. A right laparoscopic adrenalectomy was performed and the patient commenced on replacement hydrocortisone. Two weeks postoperatively serum DHEAS levels were unrecordable and testosterone levels had fallen to $0.8 \mathrm{nmol} / \mathrm{l}$. The patient was treated with hydrocortisone postoperatively to avoid hypoadrenalism and this was reduced and stopped over the next 4 months. The patient remains well with no signs of recurrence. Her blood pressure is now well controlled on two antihypertensive agents, although the achievement of glycaemic control has required the addition of insulin.

The resected specimen comprised a globular mass measuring $90 \mathrm{~mm}$ in diameter and weighing $340 \mathrm{~g}$. It had an intact thin fibrous capsule. The tumour had a uniform tan cut surface with small amounts of interstitial haemorrhage (Figure 1). Residual normal adrenal gland was attached to one pole of the mass. Histology showed the tumour to have a distinctive oncocytic appearance, with sheets and trabeculae of rounded cells with abundant granular eosinophilic cytoplasm and regular round nuclei (Figure 2). Mitotic figures were not identified and there was no tumour necrosis or evidence of capsular or vascular invasion. The adjacent adrenal gland was essentially normal.

Immunohistochemistry showed the tumour cells to be positive for high-molecular-weight cytokeratin, synaptophysin, vimentin and melan A. The cells were negative for epithelial membrane antigen, 